\title{
APROVEITAMENTO DO CAMU-CAMU (Myrciaria dubia) PARA PRODUÇÃO DE BEBIDA ALCOÓLICA FERMENTADA
}

\author{
Roberto Nobuyuki MAEDA \& Jerusa Souza ANDRADE
}

\begin{abstract}
RESUMO - O elevado teor de ácido ascórbico no camu-camu (Myrciaria dubia McVaugh, Myrtaceae) desperta o interesse de extrativistas, agricultores e consumidores, e leva à necessidade de desenvolvimento de tecnologias adequadas para produção em terra firme e aproveitamento industrial do fruto. Este trabalho teve por objetivo verificar a adequação do camu-camu para a produção de bebida alcoólica fermentada, assim como o efeito do branqueamento do fruto e da incorporação da casca à polpa nas características nutricionais e sensoriais da bebida. Os frutos foram separados em quatro lotes, sendo dois branqueados $\left(90^{\circ} \mathrm{C}\right.$ por $\left.7 \mathrm{~min}\right)$. Após a despolpa, as cascas de um lote de cada tratamento (com e sem branqueamento) foram incorporadas às respectivas polpas e avaliadas quanto à composição química (umidade, $\mathrm{pH}$, acidez, sólidos solúveis, açúcares, ácido ascórbico, compostos fenólicos, antocianinas e flavonóides). Após a correção do mosto com açúcar, pasteurização, fermentação (25 dias), trasfega, pasteurização $\left(70{ }^{\circ} \mathrm{C}\right.$ por 15 $\mathrm{min}$ ), filtragem e clarificação, as bebidas foram avaliadas quanto a composição química, edulcoradas e submetidas à análise sensorial. O branqueamento reduziu a concentração de ácido ascórbico das polpas (33\%) e a agregação da casca aumentou os teores de matéria seca (39\% polpa), ácido ascórbico (33\% na polpa, $23 \%$ no mosto e $50 \%$ na bebida) e fenólicos (50\% bebida). O perfil sensorial e a aceitabilidade sugerem que o camu-camu é adequado para a produção de bebida alcoólica fermentada e que a agregação da casca à polpa contribuiu positivamente para a aceitabilidade (6,7 com casca e 6,2 sem casca, na escala de 9 pontos). As bebidas apresentaram flavor característico do fruto, limpidez, coloração vermelho-laranjada e sabor agradável.
\end{abstract}

Palavras-chave: Fruto da Amazônia, composição química, Saccharomyces cerevisiae, fermentação, análise sensorial

\section{The use of camu-camu (Myrciaria dubia) for the production of a fermented alcoholic beverage}

\begin{abstract}
The high levels of ascorbic acid in camu-camu (Myrciaria dubia McVaugh, Myrtaceae) have stimulated interest of extractivists, farmers and consumers. This has led to a need to develop adequate technology for it's production on non-flooded land and the industrial use of this fruit. This study had as its main objective to verify if camu-camu is adequate for the production of fermented alcoholic beverages, measuring the effect of blanching the fruit and the incorporation of the fruit peel with the fruit pulp on the nutritional and sensory characteristics of the drink. The fruits were separated into 4 groups, two being blanched ( $90{ }^{\circ} \mathrm{C}$ for 7 minutes). After the pulp was removed, the peels of one group from each blanching treatment were incorporated into the respective pulps and their chemical composition evaluated. After sugar correction of the must, pasteurisation, fermentation ( 25 days), decanting, pasteurisation $\left(70{ }^{\circ} \mathrm{C}\right.$ for 15 minutes), filtering and clarification, the beverages were evaluated as to their chemical composition, sweetened and submitted to sensory analysis. Blanching reduced the concentration of ascorbic acid in the pulps $(33 \%)$ and the addition of the peel increased the amount of dry matter (39\% in pulp), ascorbic acid (33\% in pulp, $23 \%$ in must and $50 \%$ in drink) and phenolic compounds (50\% in drink). The sensory profile and acceptability suggest that camu-camu is adequate for the production of fermented alcoholic beverages and that the addition of the peel to the pulp contributes positively to it's acceptability (6.7 with versus 6.2 without, of 9 points
\end{abstract}

Instituto Nacional de Pesquisas da Amazônia-INPA/CPTA, Caixa postal - 478, CEP 69011-970 Manaus-AM 
possible). The beverage had flavour characteristic of the fruit, a orangish-red color and agreeable taste.

Key-words: Amazonian fruit, chemical composition, Saccharomyces cerevisiae, fermentation, sensory evaluation.

\section{INTRODUÇÃO}

A uva é a matéria-prima utilizada na produção do vinho, uma bebida alcoólica fermentada das mais antigas e de consumo mundial. Teoricamente, qualquer fruto ou vegetal que contenha umidade, açúcar e nutrientes para as leveduras pode ser utilizado como matéria-prima para a produção de bebidas alcoólicas fermentadas (Martinelli Filho, 1983). Além da uva, bebidas alcoólicas fermentadas de frutos como maçã (Pyrus malus), pêra (Pyrus communis), cereja (Prunus cerasus), morango (Fragaria xananassa), framboesa (Rubus idaeus), laranja (Citrus sinensis), groselha (Ribes rubrum) e outros, são também produzidas e consumidas em vários países (Vogt \& Jakob, 1986; Kolb, 2002). No Brasil, Dias et al. (2000) realizou estudos com diferentes espécies de frutos tropicais como cajá (Spondias mombin), cacau (Theobroma cacao), siriguela (Spondias purpurea) e graviola (Annona muricata) alcançando resultados promissores, demonstrando dessa forma mais uma opção para o aproveitamento destes frutos tropicais. Outro fruto tropical que pode ser usado para produção de bebidas alcoólicas fermentadas é o camu-camu, Myrciaria dubia McVaugh (Maeda \& Andrade, 2000), um fruto pertencente à família Myrtaceae, de ocorrência silvestre em regiões alagadas, ou periodicamente alagadas, como as margens de rios e lagos.

A Legislação Brasileira para bebidas alcoólicas fermentadas, Decretos $\mathrm{n}^{\circ} 73.267$, de 06 de dezembro de 1973, e 96.354, de 18 de julho de 1988, que regulamentavam a padronização, a classificação, o registro, a inspeção, a produção e a fiscalização de bebidas, permitiam a adição de açúcar em concentrações no máximo igual ao encontrado nos frutos. No entanto, o Decreto $\mathrm{n}^{\mathrm{o}} 2.314 / 97$, que regulamenta a Lei $n^{\circ} 8.918$, de 14 de julho de 1994, limita a concentração de sacarose apenas para sidra, o que permite estudos tecnológicos para o aproveitamento de frutos com baixas concentrações de açúcares, para elaboração de bebidas alcoólicas fermentadas. Entretanto a adição de sacarose em concentrações superiores aos do fruto pode ocasionar perda de suas características, levando à necessidade de estudos em relação aos limites para correção do açúcar para cada espécie utilizada para elaboração de bebida alcoólica fermentada. No presente estudo, levou-se em consideração o aspecto tecnológico para elaboração de bebida alcoólica fermentada de camu-camu como alternativa de aproveitamento do fruto.

Na Amazônia peruana Alvarado-Vertiz (1969) e Gutierrez-Ruiz (1969) relataram o consumo do camu-camu na forma de sucos, geléias, sorvetes e balas. No Brasil esse fruto era utilizado somente pelos índios e caboclos como isca para a pesca, porém, com a divulgação da alta concentração de ácido ascórbico (Andrade, 1991), aumentou-se o interesse para o consumo, incentivando o comércio, extrativismo e estudos relacionados à adaptação e formas de propagação em terra firme. Apesar do aumento da oferta, o sabor amargo e elevada acidez da polpa restringe o consumo, justificando o desenvolvimento de tecnologias para minimizar esse sabor e desenvolver formas de aproveitamento industrial. $\mathrm{O}$ fruto apresenta características desejáveis para a produção de licor (Pinto, 1998) e também para bebida alcoólica fermentada, tais como: atraente coloração vermelha (Silva \& Andrade, 1997); sabor acentuado e excelente aroma (Gutierrez-Ruiz, 1969); pH ácido, alto rendimento em polpa (Andrade, 1991; Andrade et al., 1991; Caldas, 1996). Assim, este trabalho teve por objetivo verificar a adequação do fruto para a produção de bebida alcoólica fermentada, bem como o efeito do branqueamento do fruto e da incorporação da casca à polpa nas características sensoriais da bebida. 


\section{MATERIAL E MÉTODOS}

Utilizou-se frutos maduros procedentes de populações naturais localizadas em área de várzea no Município de Careiro-Amazonas, e adquiridos no Mercado Adolpho Lisboa em Manaus, AM. Os frutos foram selecionados, descartando-se os amassados e com grau de maturação avançado, e separados em quatro lotes, sendo cada lote em três repetições (sub lotes). Após a pesagem dos lotes, dois foram submetidos ao branqueamento por imersão em água a $90{ }^{\circ} \mathrm{C}$ durante 7 minutos e ao resfriamento imediato em água com gelo. Esse branqueamento foi realizado por que Caldas (1996) afirmou que o tratamento térmico antes da despolpa reduz o sabor amargo da polpa, característica que dificulta seu consumo na forma de suco. A despolpa foi feita por prensagem manual, utilizando-se tela de nylon com malha de $1 \mathrm{~mm}$, obtendo-se uma massa homogênea. As cascas de um lote de cada tratamento (com e sem branqueamento) retidas na malha de prensagem foram manualmente separadas das sementes e incorporadas às respectivas polpas. Após a pesagem as polpas foram codificadas em B (polpa de fruto branqueado), SB (polpa de fruto não branqueado), $\mathrm{BC}$ (polpa de fruto branqueado e incorporada de casca) e SBC (polpa de fruto sem branqueamento e incorporada de casca).

Para a caracterização química das polpas foram retiradas amostras (100 g de cada tratamento) e submetidas às seguintes análises: umidade (dessecação a $65^{\circ} \mathrm{C}$ em estufa com circulação de ar forçada); $\mathrm{pH}$ (potenciômetro); acidez (titulação com NaOH $0,1 \mathrm{~N}$ e fenolftaleína como indicador); sólidos solúveis (refratometria); açúcares redutores pelo método de Somogy Nelson (Southgate, 1991); ácido ascórbico por extração com ácido oxálico a $0,5 \%$ e titulação com 2,6-diclorofenolindofenol (Ranganna, 1986); compostos fenólicos (Schanderl, 1970); antocianinas e flavonóides totais (Lees \& Francis, 1972). O rendimento em polpa foi calculado com base nos dados de pesagens dos frutos e das polpas obtidas. A relação Brix/acidez da polpa foi calculada, considerando-se os resultados dos sólidos solúveis e da acidez titulável (expressa em percentagem de ácido cítrico).
O restante das polpas foi utilizado para a produção das bebidas. Para alcançar o teor alcoólico de $12{ }^{\circ} \mathrm{GL}$ na bebida final, o mosto foi corrigido com a adição de açúcar, cuja quantidade foi calculada considerando-se os resultados de açúcares, da quantidade de polpa a ser fermentada e do volume de bebida (Maeda, 1999). O açúcar foi dissolvido em água, aquecido até a fervura $\left(97^{\circ} \mathrm{C}\right)$, adicionado da polpa e mantida nesta temperatura por cerca de cinco minutos para pasteurizar a polpa e xarope, e assim, reduzir a flora contaminante, e consequentemente, a competição com as outras leveduras.

A mistura de xarope e polpa foi resfriada por imersão da panela em banho de água com gelo, e transferida imediatamente para a cuba de fermentação (garrafões de vidro com capacidade para 20 litros), adicionada de água potável fria, previamente fervida, até completar 18 litros e homogeneizada. Para a hidratação da levedura Saccharomyces cerevisiae (fermento seco Fleischmann), foram adicionados $20 \mathrm{~g}$ de levedura em dois litros do mosto, mantidos em repouso, com agitações ocasionais, por cerca de duas horas e retornados a cuba. A fermentação foi conduzida por 25 dias, em ambiente protegido da luminosidade e com temperatura controlada de $25 \pm 1{ }^{\circ} \mathrm{C}$.

Após a trasfega, a bebida foi acondicionada em garrafas de vidro, pasteurizada a $70{ }^{\circ} \mathrm{C}$ por 15 minutos, resfriada e filtrada (utilizando-se papel de filtro $\mathrm{n}^{\circ} 16 \mathrm{e}$ bomba de vácuo). A clarificação e maturação da bebida foram obtidas pelo armazenamento (com trasfegas a cada três meses) durante seis meses em ambiente escuro e com temperatura controlada de $25^{\circ} \mathrm{C}$.

Após seis meses as bebidas (antes da edulcoração) foram avaliadas quanto ao teor de ácido ascórbico (Ranganna, 1986), acidez total (titulação com solução de $\mathrm{NaOH} 0,1 \mathrm{~N}$, fenolftaleína como indicador e os resultados expressos em percentagem de ácido cítrico), açúcares redutores (Somogy Nelson segundo Southgate, 1991) e compostos fenólicos (Schanderl, 1970), com os resultados expressos em $100 \mathrm{~mL}$ de bebida. O grau alcoólico, após destilação, foi determinado com alcoômetro de Gay-Lussac (Instituto Adolfo Lutz, 1995). O rendimento alcoólico foi calculado com base na 
quantidade em gramas de açúcar consumido e álcool produzido e os resultados foram expressos em $\%(\mathrm{p} / \mathrm{p})$. A eficiência fermentativa foi calculada pela relação entre o rendimento alcoólico e o valor estequiométrico teórico da conversão de açúcar em álcool (Aquarone \& Zacanaro Júnior, 1983; Bortolini, 2001).

Imediatamente após as análises químicas, as bebidas foram submetidas a testes sensorias preliminares, nos quais 15 entre 20 provadores apontaram a necessidade de edulcoração para suavizar o sabor. Com base nas observações dos provadores as bebidas foram adicionadas de açúcar até atingir a concentração final de 8,5 ${ }^{\circ}$ Brix. As bebidas edulcoradas foram submetidas à análise sensorial utilizando-se a escala hedônica estruturada de nove pontos e um grupo de 27 provadores, e os dados foram avaliados por análise de variância e teste de Tukey (Monteiro, 1984).

\section{RESULTADOS E DISCUSSÃO}

O camu-camu é um fruto com elevado teor de umidade, e consequentemente, baixa concentração de matéria seca. A incorporação da casca à polpa aumentou o rendimento em $30,8 \%$ dos frutos branqueados e $35,1 \%$ dos frutos não branqueados (Tabela 1 ), contribuindo para a disponibilidade de material a ser utilizada no preparo do mosto. $\mathrm{O}$ alto rendimento da fração industrializável é uma característica desejável nos processos tecnológicos para elaboração de produtos. A polpa incorporada de casca apresentou rendimento elevado, refletindo no valor tecnológico, e consequentemente, no valor econômico do fruto. Como a casca do camu-camu apresenta maior concentração de ácido ascórbico, pigmentos e compostos fenólicos (Caldas, 1996), sua utilização no preparo de licor (Pinto, 1998) e bebida alcoólica fermentada é desejável (Maeda, 1999). Maior concentração desses componentes pode ser extraída no processo de maceração do licor ou incorporada à bebida no processo de fermentação da bebida alcoólica, e o resíduo de casca não altera as características das bebidas por ser descartado no processo normal de decantação e filtração.
O camu-camu apresentou acidez elevada e baixo pH (Tabela 1). Com exceção da polpa $\mathrm{B}$, não foram observadas variações significativas entre os tratamentos. Andrade (1991) reportou que o baixo $\mathrm{pH}$ apresentado pelo camu-camu é uma característica tecnológica desejável na delimitação do tempo e do tipo de tratamento térmico para o processamento industrial. Os valores de acidez mostraram-se superiores a média $(2,7 \%)$ encontrada em frutos de terra firme e várzea (Andrade, 1991; Silva \& Andrade, 1997), indicando variações em função da procedência dos frutos. A elevada concentração de ácidos das polpas foi suficiente para manter a acidez no mosto dentro da faixa desejável para produção de bebida alcoólica fermentada.

Variações irregulares foram observadas nos açúcares redutores entre os tratamentos empregados (Tabela 1). Sem as cascas, as polpas apresentaram variações mais acentuadas nas concentrações de açúcares redutores. Esses açúcares apresentaram concentrações inferiores às detectadas por Silva \& Andrade (1997). Como os açúcares constituem-se em substrato para a produção de álcool, a baixa concentração presente na polpa foi corrigida com a adição de açúcar no mosto.

De acordo com Kolb (2002), mosto de morango sem a adição de açúcar produz um vinho com baixa capacidade de armazenamento. Então, para se produzir um vinho de mesa é imprescindível a adição de açúcar em mostos não diluídos, na concentração de 150 a $200 \mathrm{~g}$ por litro de mosto. Devido ao alto custo dos frutos de morango, recomenda-se a adição de água ao mosto quando a produção é em pequena escala, e neste caso, a quantidade de açúcar deverá ser em função da diluição e do grau alcoólico desejado. Partindo de suco de laranja com 9,5 ${ }^{\circ}$ Brix, Corazza et al. (2001) fizeram a correção com açúcar elevando a concentração para $26,5^{\circ}$ Brix, obtendo um vinho de laranja com $10,6^{\circ} \mathrm{GL}$ com excelente sabor e qualidade sensorial.

Os sólidos solúveis do camu-camu, compostos em sua maioria pelos componentes ácidos do fruto, apresentaram comportamento similar ao apresentado pela acidez titulável. Com exceção da polpa $\mathrm{B}$, as demais não diferiram quanto a relação Brix/acidez, as quais 
ressaltaram o baixo grau de doçura do fruto.

A posição de destaque do camu-camu dentre os frutos da Amazônia decorre da alta concentração de ácido ascórbico. Devido sua instabilidade ao calor, o branqueamento ocasionou perda de 33,3\% em polpas sem casca e $34,4 \%$ em polpas com a casca incorporadas. Em função da casca deter concentrações mais elevadas de ácido ascórbico (Andrade, 1991), sua incorporação à polpa contribuiu positivamente, elevando os teores em $23,8 \%$ nas polpas de frutos branqueados e $25,0 \%$ em polpas de frutos não branqueados.
Sem o branqueamento do fruto e com a incorporação de casca, as polpas apresentaram concentrações mais elevadas de compostos fenólicos, antocianinas e flavonóides totais (Tabela 1). Observou-se ainda que o branqueamento dos frutos reduziu a concentração desses compostos. A importância desses compostos decorre da ação de alguns fenólicos e das antocianinas no sabor e coloração da bebida, assim como da propriedade antioxidante dos flavonóides. Teores mais elevados desses constituintes foram detectados em frutos de camu-camu

Tabela 1- Rendimento e composição química das polpas de camu-camu utilizadas para a produção da bebida alcoólica fermentada. B - polpa de fruto branqueado, SB - polpa de fruto não branqueado, $\mathrm{BC}$ - polpa de fruto branqueado + casca, $\mathrm{SBC}$ - polpa de fruto não branqueado + casca.

\begin{tabular}{lcccc}
\hline COMPOSIÇÃO & $\mathrm{B}$ & $\mathrm{SB}$ & $\mathrm{BC}$ & $\mathrm{SBC}$ \\
\hline Rendimento em polpa (\%) & 56,9 & 56,2 & 82,20 & 86,6 \\
Umidade (\%) & $94,35 \pm 0,70$ & $92,75 \pm 0,57$ & $90,55 \pm 1,01$ & $89,89 \pm 1,10$ \\
$\mathrm{pH}$ & $3,20 \pm 0,10$ & $2,70 \pm 0,15$ & $2,65 \pm 0,10$ & $2,67 \pm 0,12$ \\
Acidez (\% em ácido cítrico) & $2,91 \pm 0,04$ & $4,40 \pm 0,16$ & $4,31 \pm 0,03$ & $4,10 \pm 0,03$ \\
Sólidos solúveis ('Brix) & $5,57 \pm 0,30$ & $6,77 \pm 0,20$ & $7,31 \pm 0,30$ & $6,78 \pm 0,40$ \\
Relação Brix/acidez & 1,91 & 1,54 & 1,70 & 1,65 \\
Açúcares redutores (\%) & $1,40 \pm 0,19$ & $2,05 \pm 0,12$ & $1,71 \pm 0,13$ & $1,64 \pm 0,07$ \\
Ácido ascórbico (\%) & $0,80 \pm 0,05$ & $1,20 \pm 0,06$ & $1,05 \pm 0,02$ & $1,60 \pm 0,08$ \\
Compostos fenólicos (mg/100 g) & $983,3 \pm 4,24$ & $978,4 \pm 5,20$ & $975,9 \pm 4,20$ & $993,1 \pm 5,50$ \\
Antocianinas totais (mg/100 g) & $1,92 \pm 0,23$ & $2,12 \pm 0,08$ & $1,90 \pm 0,20$ & $3,05 \pm 0,93$ \\
Flavonóides totais (mg/100 g) & $16,93 \pm 0,03$ & $16,38 \pm 0,11$ & $17,27 \pm 0,10$ & $19,12 \pm 0,06$ \\
\hline
\end{tabular}

procedentes de cultivo em várzea (Silveira, 1998).

O meio de fermentação apresentou coloração vermelha, característica do camucamu. Como os pigmentos estão presentes na casca, sua agregação à polpa contribuiu para coloração mais acentuada nos tratamentos BC e SBC. No entanto, em função da instabilidade das antocianinas, no início da fermentação tumultuosa observou-se, em todos os tratamentos, a degradação desses pigmentos, implicando-se em mudança gradativa da cor vermelho-púrpura para vermelho-alaranjada.

O camu-camu apresenta características desejáveis para a produção de bebida alcoólica fermentada, destacando-se o alto rendimento em bebida, que atingiu valores de 85 a $90 \%$ em relação ao volume de mosto. Nas condições experimentais, os resultados permitem estimar que, a partir de um $1 \mathrm{~kg}$ de fruto, obtém-se cerca de 8,5 litros de bebida alcoólica. O processo fermentativo apresentou um bom rendimento alcoólico $(44,5 \%)$ e boa eficiência fermentativa $(87,1 \%)$, mostrando assim, as boas condições do meio e da levedura inoculada. Observou-se também, que os diferentes tratamentos de obtenção das polpas não tiveram influência sobre estes parâmetros.

As composições químicas das bebidas (Tabela 2) mostraram que o branqueamento do fruto, assim como a incorporação da casca não modificou significativamente algumas características, tais como $\mathrm{pH}$, sólidos solúveis e graduação alcoólica. Valores mais elevados de 
acidez foram detectados nas bebidas produzidas com polpas incorporadas de casca. Observou-se que os baixos valores de $\mathrm{pH}$ encontrados nas polpas também foram detectados nas bebidas e sem efeito dos tratamentos empregados para a obtenção das polpas. Quanto a graduação alcoólica, o mosto foi corrigido para a obtenção de $12 \%$ e o valor de $11,0 \%$ obtido pode ser considerado satisfatório.

Variações significativas nas concentrações de compostos fenólicos foram observadas nas bebidas em função dos tratamentos empregados (Tabela 2). As bebidas obtidas com polpas de frutos branqueados apresentaram valores mais elevados de compostos fenólicos. Da mesma forma a incorporação da casca implicou em aumento desses compostos nas bebidas. $\mathrm{O}$ camu-camu é um fruto que apresenta alta concentração de compostos fenólicos, na faixa de 1,37 (Silva, 1997) a 2,11\% (Andrade, 1991), os quais podem ser relacionados com as características sensoriais de amargor e adstringência do fruto (Andrade, 1991), influenciando assim, no sabor da bebida. Os valores obtidos nas bebidas estão de acordo com os reportados (100 a $300 \mathrm{mg} / \mathrm{L})$

Tabela 2 - Efeito do branqueamento e da agregação da casca em alguns componentes da bebida alcoólica fermentada de camu-camu, após seis meses de armazenamento. B - polpa de fruto branqueado, SB - polpa de fruto não branqueado, $\mathrm{BC}-$ polpa de fruto branqueado + casca, $\mathrm{SBC}$ - polpa de fruto não branqueado + casca.

\begin{tabular}{lcccc}
\hline COMPONENTES & $\mathrm{B}$ & $\mathrm{SB}$ & $\mathrm{BC}$ & $\mathrm{SBC}$ \\
\hline Acidez (\% em ácido cítrico) & 0,47 & 0,49 & 0,55 & 0,55 \\
Açúcares redutores $(\mathrm{g} / 100 \mathrm{~mL})$ & 1,03 & 1,16 & 1,10 & 1,07 \\
$\mathrm{pH}$ & 2,70 & 2,70 & 2,70 & 2,70 \\
Sólidos solúveis & 4,50 & 4,50 & 4,50 & 4,50 \\
Álcool ( $\left.{ }^{\circ} \mathrm{GL}\right)$ & 11,00 & 11,00 & 11,00 & 11,00 \\
Compostos fenólicos $(\mathrm{mg} / 100 \mathrm{~mL})$ & 140,44 & 81,62 & 180,88 & 123,90 \\
\hline
\end{tabular}

para vinhos tintos (Bauerfeind \& Pinkert, 1970).

A degradação do ácido ascórbico nas polpas, decorrente do branqueamento do fruto, foi compensada pela agregação da casca, contribuindo assim para manter mais elevados os níveis de ácido ascórbico nos tratamentos $\mathrm{BC}$ e SBC (Figura 1). Dentre os fatores responsáveis pela redução do ácido ascórbico, destacam-se a degradação pelo calor durante o branqueamento dos frutos e pasteurização do mosto, a diluição pela adição de água no preparo do mosto e a degradação durante a fermentação.

Apesar de constituir-se em processos drásticos para a estabilidade do ácido ascórbico, sua alta concentração inicial contribuiu para que a concentração na bebida possa ser considerada ainda relativamente elevada. De acordo com Bauerfeind \& Pinkert (1970), a adição de ácido ascórbico ao vinho na proporção de 25 a $100 \mathrm{mg} / \mathrm{L}$ ajuda a estabilizar as reações de oxidação-redução com efeito favorável no sabor, aroma e limpidez. Desta forma, o teor de ácido ascórbico ainda presente na bebida pode ser considerado satisfatório e com implicações favoráveis no valor nutricional e nas características de sabor, aroma e limpidez.

Os resultados da análise sensorial mostram que a bebida SBC apresentou maiores notas e que as bebidas $\mathrm{B}, \mathrm{SB}$ e BC não diferiram entre si ao nível de $5 \%$ de significância (Tabela 3). Estes resultados podem indicar o efeito negativo do branqueamento, nas condições testadas, no flavor das bebidas. Quanto a aceitabilidade, a bebida SBC apresentou valor 
de 79,05\%, enquanto as demais apresentaram $69,2,70,1$ e 70,9 para as bebidas B, SB e BC, respectivamente. A característica levemente adstringente das bebidas foi suavizada pela adição de açúcar. De acordo com comentários dos provadores, as bebidas preparadas a partir de frutos branqueados e as bebidas em que foram utilizadas polpa sem incorporação da casca apresentaram sabor mais "fraco", enquanto a bebida feita a partir de polpa de frutos não branqueados e que tiveram a casca incorporada à polpa, apresentou sabor e aroma mais acentuado.

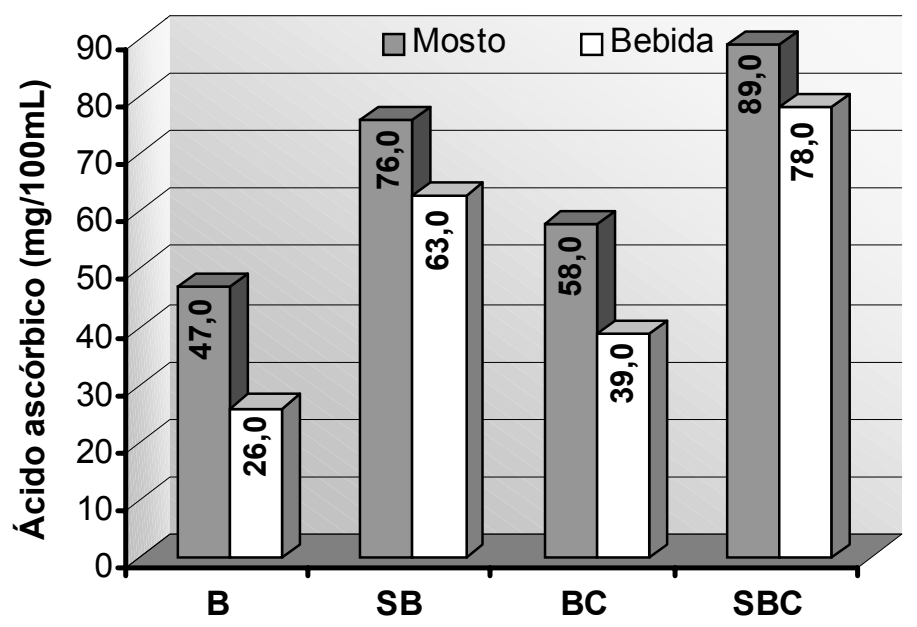

Figura 1 - Teores de ácido ascórbico nos mostos e nas bebidas alcoólicas de camu-camu. B polpa de fruto branqueado, $\mathrm{SB}$ - polpa de fruto não branqueado, $\mathrm{BC}$ - polpa de fruto branqueado + casca, SBC - polpa de fruto não branqueado + casca.

Tabela 3 - Notas atribuídas às bebidas alcoólicas de camu-camu pelos 27 provadores na análise sensorial. $\mathrm{B}$ - polpa de fruto branqueado, $\mathrm{SB}$ - polpa de fruto não branqueado, $\mathrm{BC}$ - polpa de fruto branqueado + casca, SBC - polpa de fruto não branqueado + casca.

\begin{tabular}{cccc}
\hline Tratamentos & Notas & \multicolumn{2}{c}{ Freqüência de respostas $(\%)$} \\
\cline { 3 - 4 } & (Média \pm DP) & Valores $\leq 4$ & Valores $\geq 6$ \\
B & $6,15 b \pm 1,99$ & 14,81 & 62,96 \\
SB & $6,33 b \pm 1,90$ & 18,52 & 70,37 \\
BC & $6,37 b \pm 1,96$ & 18,52 & 77,78 \\
SBC & $7,04 \mathrm{a} \pm 1,81$ & 7,41 & 85,18 \\
d.m.s. & 0,325 & & \\
\hline
\end{tabular}

Médias seguidas pelas mesmas letras não diferem a $5 \%$ de probabilidade no teste de Tukey.

\section{CONCLUSÃO}

O camu-camu mostrou-se adequado para a produção de bebida alcoólica fermentada e a agregação da casca à polpa contribuiu positivamente para a aceitabilidade da bebida. O branqueamento e a remoção da casca ocasionaram um efeito negativo, reduzindo a aceitabilidade de $79 \%$ para aproximadamente $70 \%$. 


\section{BIBLIOGRAFIA CITADA}

Alvarado-Vertiz, M.A. 1969. Posibilidades del cultivo del camu-camu en el Peru, Myrciaria dubia. Monografia, Pontifícia Universidad Católica del Peru, Lima, Peru, 51p.

Andrade, J.S. 1991. Curvas de maturação e características nutricionais do camu-camu (Myrciaria dubia (HBK) McVaugh) cultivado em terra firme na Amazônia Central Brasileira. Tese de Doutorado, Universidade de Campinas, Campinas, São Paulo, 177 p.

Andrade, J.S.; Galeazzi, M.A.M.; Aragão, C.G.; Chávez Flores, W.B. 1991. Valor nutricional do camu-camu (Myrciaria dubia (H.B.K.) McVaugh) cultivado em terra firme na Amazônia central. Revista Brasileira de Fruticultura, 13(2): 307-311.

Aquarone, E.; Zacanaro Júnior, O. 1983. Vinagres. In: Aquarone, E.; Lima, U.A; Borzani, W. Alimentos e bebidas produzidas por fermentação. Ed. Edgard Blücher, São Paulo, São Paulo, v. $5,227 \mathrm{p}$.

Bauernfeind, J. C.; Pinkert, D. M. 1970. Food processing with added ascorbic acid, $A d$ vances in Food Research, 18: 219-315.

Bortolini, F.; Santanna, E.S.; Torres, R.C. 2001. Comportamento das fermentações alcoólica e acética de sucos de kiwi (Actinidia deliciosa): composição dos mostos e métodos de fermentação acética. Ciência e Tecnologia de Alimentos, 21(2): 236-243.

Caldas, M.L.M. 1996. Efeito dos métodos de despolpa e do congelamento na conservação da polpa de camu-camu (Myrciaria dubia McVaugh). Monografia, Faculdade de Ciências Agrárias, Universidade Federal do Amazonas, Manaus, Amazonas, $63 \mathrm{p}$.

Corazza, M.L.; Rodríguez, D.G.; Nozaki, J. 2001. Preparação e caracterização do vinho de laranja. Química Nova, 24(4): 449-452.

Dias, E.R.; Schwan, R.F.; Carvalho, E.P.; Lima, L.C.O. 2000. Elaboração de bebida alcoólica fermentada a partir de frutas tropicais. Livro de Resumos do XVII Congresso Brasileiro de Ciência e Tecnologia de Alimentos. Sociedade Brasileira de Ciência e Tecnologia de Alimentos, Fortaleza, Ceará, v. 3, p. 11.68.

Gutierrez-Ruiz, A. 1969. Especies frutales nativas de la selva del Perú: estudio botánico y de propagación por semillas. Monografia, Universidad Nacional Agraria La Molina, La Molina, Peru, 91 p.

Instituto Adolfo Lutz. 1985. Normas analiticas do Instituto Adolfo Lutz: Métodos químicos e físicos para análise de alimentos. Instituto Adolfo Lutz, São Paulo, v. 1, 533 p.

Kolb, E. 2002. Vinos de frutas. Ed. Acribia, Zaragoza, Espanha, 280 p.

Lees, D.H.; Francis, F.J. 1972. Standardization of pigment analyses in cranberries. Hort. Science, 7(1):83-84.

Maeda, R.N. 1999. Adequação tecnológica do сатu-camu (Myrciaria dubia McVaugh) para produção de vinho. Monografia, Universidade Federal do Amazonas, Manaus, Amazonas, 58p.

Maeda, R.N.; Andrade, J.S. 2000. Obtenção de bebida alcoólica de frutos de camu-camu (Myrciaria dubia McVaugh). Livro de Resumos do XVII Congresso Brasileiro de Ciência e Tecnologia de Alimentos, Sociedade Brasileira de Ciência e Tecnologia de Alimentos, Fortaleza, Ceará, v. 4 , p. 11.145 .

Martinelli Filho, A. 1983. Tecnologia de vinhos $e$ vinagres de frutas. Departamento de Tecnologia Rural da ESALQ/USP. Piracicaba, São Paulo, 130 p.

Monteiro, C.L.B. 1984. Técnicas de avaliação sensorial. $2^{\text {a }}$ ed., Universidade Federal do Paraná, Curitiba, Paraná, 184 p.

Pinto, J.R.S. 1998. Adequação tecnológica do camu-camu (Myrciaria dubia McVaugh): produção de licores. Monografia, Universidade Federal do Amazonas, Manaus, Amazonas, 39p.

Ranganna, S. 1986. Analysis and quality control for fruit and vegetable products. Tata McGraw-Hill Publishing, New Delhi, $1112 \mathrm{p}$. 
Schanderl, S.H. 1970. Tannins and related phenolics. In: Joslyn, M.A. Methods in Food Analysis. Ed. Academic Press inc., New York, p. 701-725.

Silva, C.T.C. 1997. Conservação pós-colheita de camu-camu (Myrciaria dubia (H.B K.) McVaugh) pelo uso de atmosfera modificada e refrigeração. Dissertação de mestrado, Universidade Federal do Amazonas, Manaus, Amazonas, 112 p.

Silva, C.T.C.; Andrade, J.S. 1997. Post harvest modifications in camu-camu fruit (Myrciaria dubia McVaugh) in response to stage of maturation and modi- fied atmosphere. Acta Horticulture, 452: 23-26.

Silveira, J.S. 1998. Efeito do estádio de maturação na conservação pós-colheita de camu-camu (Myrciaria dubia McVaugh). Dissertação de mestrado, Universidade Federal do Amazonas, Manaus, Amazonas, $115 \mathrm{p}$.

Southgate, D. A. T. 1991 Determination of foods carbohydrates. Elservier Applied Science, London, 232p.

Vogt, E.; Jakob, L. 1986. El vino: obtención, elaboración y análisis. Ed. Acribia, Zaragoza, 292 p. 
